\title{
Nonlinear Simulation of Edge-Localized Mode in Spherical Tokamak
}

\author{
Naoki Mizuguchi* Member \\ Riaz Khan** Non-member \\ Takaya Hayashi* Non-member
}

\begin{abstract}
Numerical simulation which is based on the nonlinear resistive magnetohydrodynamic(MHD) model is executed to reveal the dynamics of the edge-localized modes(ELMs) in the spherical tokamak(ST) plasma. Characteristic features of the ELMs, such as the formation of the filaments, the toroidal localization of the mode structures, and the separation of plasmoid from the bulk, have been successfully reproduced. These processes are found to be the consequence of the nonlinear growth of the ballooning mode under the MHD assumptions. The simulation results are compared with the experimental observations in Mega-Amp Spherical Tokamak(MAST), and show good agreement.
\end{abstract}

Keywords: ELM, MHD, simulation, filament, blob

\section{Introduction}

The edge-localized mode(ELM) is an instability which is observed repetitively near the edge of the torus plasma. Experimentally, ELMs are often observed in some H-mode operations of tokamak and are categorized into several types phenomenologically. In some ELM types the instability is thought to be related to the pressure-driven ballooning mode.

In view of the confinement of plasma the ELM degrades the confinement property in that part of the stored energy is lost through the instabilities. However, ELMs are not necessarily harmful for better confinement because the particle control by the ELMs is compatible with the H-mode operation. Therefore, it is fruitful to understand the physical mechanisms of the ELM.

In some experiment, the ELM is not only observed by the edge fluctuation measurements, but also analyzed by using a camera. In particular, in Mega-Amp Spherical Tokamak(MAST) device ${ }^{(1)}$ the overall structure of the ELM activity has been observed. The camera image shows a belt-like structures emerges helically along the magnetic field lines. These structures are called "filaments" or "blobs". The spatial structure is analyzed in detail. It is shown that a plasmoid is separated radially from the main part of the torus. Such filament structures are also observed in the National Spherical Torus Experiment(NSTX).

The physical mechanism of the ELM is analyzed both experimentally and theoretically. Much interest has been focused on the intermittent nature of the blobs. There are many experimental observations of the blobs in several torus experiments ${ }^{(2)(3)}$. The intermittency is discussed in view of edge turbulence transport ${ }^{(4)}$. An-

\footnotetext{
* National Institute for Fusion Science

322-6, Oroshi-cho, Toki 509-5292

** Sokendai

322-6, Oroshi-cho, Toki 509-5292
}

other stream of the theoretical work is related to explosive phenomena in plasma such as the solar eruptions. There is an attempt to unify the theory of these phenomena by the ballooning theory ${ }^{(5)}$.

In this paper we execute a numerical simulation which is based on the nonlinear resistive magnetohydrodynamic(MHD) model to reveal the dynamics of ELMs in the spherical tokamak(ST) plasma. By using a realistic geometry and a simple model, we aim at reproducing various aspects of the phenomenon self-consistently. The simulation has been done under the same condition as reported in Ref. (6), which has shown a two-step relaxation of ST plasma. Here we focus on the early period of the simulation result, where the ballooning mode dominates the process. Detailed comparison with the experimental observations of ELMs is also mentioned.

The contents of this paper is as follows. In Sec.2, the simulation model is described. The simulation result is shown in Sec.3. Finally, we summarize the results and give the discussion in Sec.4.

\section{Simulation Model}

We use a realistic model based on the resistive MHD equations in a full toroidal geometry. The governing equations are a standard set of the nonlinear MHD equations:

$$
\begin{aligned}
& \frac{\partial \rho}{\partial t}=-\nabla \cdot(\rho \boldsymbol{v}) \\
& \frac{\partial}{\partial t}(\rho \boldsymbol{v})=-\nabla \cdot(\rho \boldsymbol{v} \boldsymbol{v})-\nabla p+\boldsymbol{j} \times \boldsymbol{B} \\
& +\mu\left(\nabla^{2} \boldsymbol{v}+\frac{1}{3} \nabla(\nabla \cdot \boldsymbol{v})\right), \\
& \frac{\partial \boldsymbol{B}}{\partial t}=-\nabla \times \boldsymbol{E} \\
& \frac{\partial p}{\partial t}=-\nabla \cdot(p \boldsymbol{v})-(\gamma-1)(p \nabla \cdot \boldsymbol{v} \\
& +\eta \boldsymbol{j}^{2}+2 \mu\left(e_{i j} e_{i j}-\frac{1}{3}(\nabla \cdot \boldsymbol{v})^{2}\right), \cdots
\end{aligned}
$$


(a)

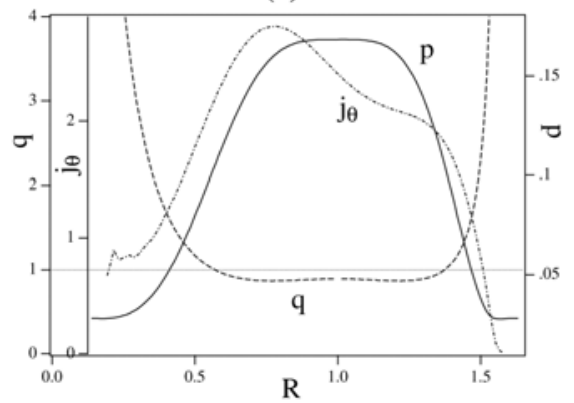

(b)

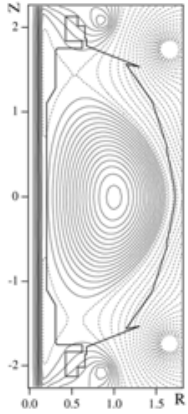

Fig. 1. (a) Radial profiles of the pressure $p$, the toroidal current $j_{\theta}$ and the safety factor $q$ for the initial condition, (b) geometry and the magnetic field structure of the initial condition

$$
\begin{aligned}
& \boldsymbol{j}=\nabla \times \boldsymbol{B}, \ldots \ldots \ldots \ldots \ldots \ldots \ldots \ldots \ldots \\
& \boldsymbol{E}=-\boldsymbol{v} \times \boldsymbol{B}+\eta \boldsymbol{j}, \\
& e_{i j}=\frac{1}{2}\left(\frac{\partial v_{i}}{\partial x_{j}}+\frac{\partial v_{j}}{\partial x_{i}}\right)
\end{aligned}
$$

The density $\rho$, the magnetic field $\boldsymbol{B}$, and the geometrical length are normalized to be one at the magnetic axis. Therefore the unit of the time $t$ corresponds to the Alfven transit time, and the velocity $\boldsymbol{v}$ is normalized by the Alfven velocity on the magnetic axis. In these equations, the explicit dissipation terms are included as the resistivity $\eta$ and the viscosity $\mu$. These terms are set to be uniform constants in this model. The heating effect of these terms are also included consistently in the evolution of the pressure $p$. The equations are solved numerically by using the fourthorder central-finite-difference scheme and a fourth-order Runge-Kutta scheme. The number of the numerical mesh is $\left(N_{r}, N_{\theta}, N_{z}\right)=(64,64,128)$, including the dead region behind the conductor wall.

The initial condition of the simulation is given from the experimental data of the NSTX. We use the reconstructed equilibrium of the shot \#10371 at $238 \mathrm{msec}$, where the central beta $\beta_{0}=28 \%$, the central safety factor $q_{0}=0.89$, and the aspect ratio $A=1.4$, as shown in Fig.1(a). One can see that these radial profiles are somewhat broad. Therefore, the pressure gradient is large in the outer region. The boundary condition is a perfect conductor, following the real shape of the machine as drawn in Fig.1(b). The simulation starts by adding tiny random perturbations in the velocity component of the initial equilibrium. Thus, the spontaneous time development of the MHD system is solved by using a supercomputer.

\section{Simulation Result}

The initial equilibrium is stable under the ideal regime, that is, $\eta$ is substantially small. Under this situation, the applied perturbations gradually dumps due to the viscosity. However, several modes become unstable with larger $\eta$. For $\eta=4 \times 10^{-5}$ (normalized),

(a)

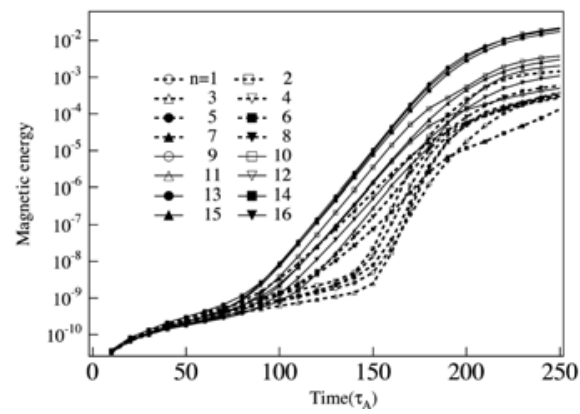

(b)

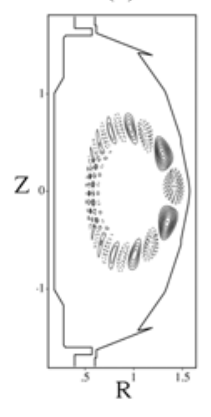

Fig. 2. (a) Time development of the magnetic energy for each toroidal Fourier mode number $n$, (b) poloidal mode structures of the $n=12$ mode: The perturbations in the plasma pressure is plotted as the contours; Solid and broken line indicates the positive and negative quantity, respectively

the time development of the magnetic energy for each toroidal Fourier mode is shown in Fig.2(a). One can see that multiple toroidal mode components are growing simultaneously, and that the most dominant ones have intermediate toroidal mode numbers such as the $n=12$ and the $n=13$. The growth rate and the most dominant mode number is sensitive to the value of $\eta$, which is set to be much larger than the realistic value of the experiments. However, the qualitative nature does not change so much, especially in the succeeding nonlinear stage. As shown in Fig.2(b), these resistive modes have the nature of the ballooning mode in that the mode structures are poloidally localized in the so-called bad-curvature region.

The three-dimensional structure of this instability is drawn in Fig.3. In this figure, the bird's-eye view picture at $t=190 \tau_{A}$, in which the system reaches the nonlinear stage, is drawn. The shape of the plasma surface is drawn by the light gray iso-pressure contour. One can see that the plasma surface is wrinkled, and that several numbers of the ridges which are equivalent to the most dominant toroidal mode number emerge along the surrounding magnetic field. Since the 'balloon' of the plasma pressure rapidly distorts the cross-field component of the magnetic field, the current is partly induced in a quite elongated region along the magnetic field. This current filament structure is shown together in Fig. 3 by the dark gray iso-pressure contour. These structures well agree with the experimental observations of MAST ${ }^{(1)}$. It should be also noted that the number of the filament is much less than that of the ridges or the dominant mode number. In this case, we can see only a few filaments in almost one side of the torus. Also in the experiments, the filaments are often observed as only a few bright narrow-width region by using a camera. This reduction mechanism can be explained by the coexistence of the multiple modes. The dominant poloidal components of each ballooning mode have almost the same helicity which is slightly above 1 , such as $m / n=13 / 12$ and $14 / 13$, where $m$ is the poloidal mode number. Therefore, these mode structures exist 


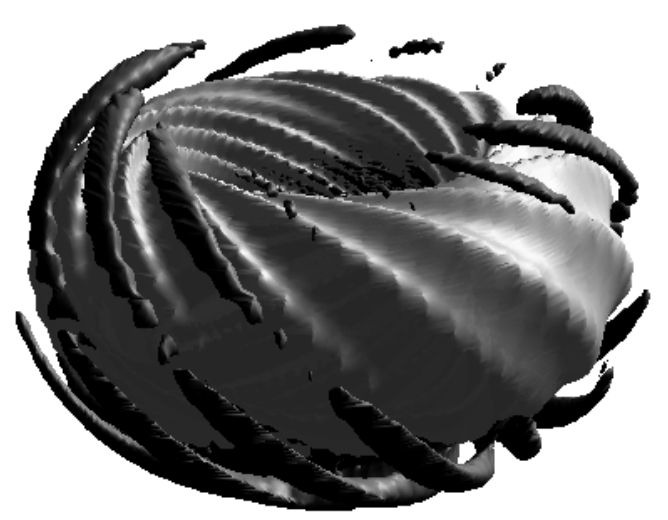

Fig. 3. Three-dimensional structure of the filaments: The dark and light gray object represents the iso-contour surfaces of the current and the plasma pressure at $t=190 \tau_{A}$, respectively; The current is plotted by the difference from the initial state, and only the toroidal component is shown (a)

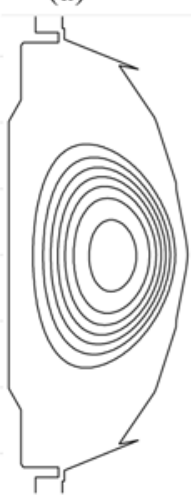

(b)

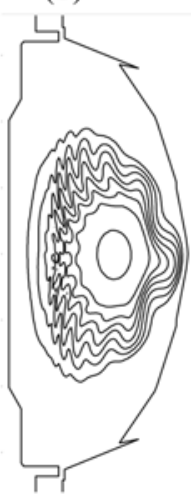

(c)

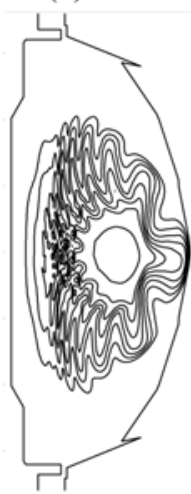

(d)

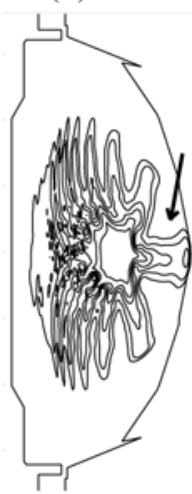

Fig. 4. Time development of the poloidal pressure profile: The time is (a) $\mathrm{t}=0,(\mathrm{~b}) 190$, (c) 210 , and (d)240 $\tau_{A}$, respectively; The contours of the net plasma pressure are plotted in each picture

very close to each other. Such situation induces the nonlinear couplings among these modes. Since the wave length of such nonlinear modes is determined by the difference between the modes concerned, low- $n$ nonlinear perturbations are superposed the overall development. Thus, the system results in a nonuniform growth of the filament-like structures in the nonlinear stage.

In Fig.4, the temporal change in the pressure profile in the poloidal cross section is shown. At the beginning of the nonlinear stage(Fig.4(b)), the plasma surface is wrinkled compared to the initial state(Fig.4(a)) due to the growth of the ballooning mode. Then the balloons grow rapidly. In particular, one of the balloons, the outermost one in the figure, changes largely in the shape. The leg of the balloon gets thin gradually(Fig.4(c)). Finally, the head of the balloon is separated from the bulk(Fig.4(d)) plasma, as indicated by the arrow in Fig.4(d). This separation process is clearly observed experimentally in MAST $^{(1)}$. In this simulation, it is revealed that such a formation of the filament structures and the separation phenomenon on ELMs can occur as a result of only the nonlinear MHD process.

\section{Summary and Discussion}

We have executed a nonlinear resistive MHD simulation in a full toroidal spherical tokamak geometry to reveal the dynamics of the filament on the ELM. The formation of the plasma blobs was found to be the consequence of the nonlinear growth of the ballooning mode. The blobs extend along the magnetic field lines and emerge in toroidally localized region. As the growth of the mode saturates, part of ridges of plasma is separated from the bulk. All of these characteristic aspects can be explained qualitatively with only the nonlinear MHD model.

The simulation results described above show some good agreement with the experimental observations. If we list the agreements, (1)the blobs are formed along the magnetic field lines, (2)the amplitude of the blobs appears non-uniformly in toroidal directions, (3)a plasmoid is ejected radially from the ridge of the blobs. Though these comparisons with our present simple model may be still rough, they provide us with valuable information to better understanding of such a complicated phenomenon. The temporal and spatial scale of an ELM is so short and steep that the MHD constraint might not be violated at the most active period like the separation process. Under such circumstance an appropriate correction may be needed. We have started such an attempt by adding the finite Larmor radius effect. It will provide more proper analysis including also the effect of the diamagnetic rotation. On the other hand, the intermittent nature of the ELM has to be treated for much longer time scale, and will require the consistent inclusion of the transport models. These are our future works.

As for the geometry effect, the basic equations (1)-(7) treats the metric operators explicitly. Moreover, the initial condition has unique features of low aspect ratio, such as high beta of the plasma. In high-beta plasma, the system tends to be more disorderd when some instabilities occure than in lower-beta plasma. All the results described above would show such behavior. However, more systematic analysis on the geometrical effects will be done in future.

Finally, we briefly introduce the sequel of this simulation. As described in Ref. (6), the growth of the ballooning mode reaches a saturation and relaxes at around $t=500 \tau_{A}$. The increased current density by the filament may enhance the magnetic reconnection between the internal and external fields on the ridges. Due to the reconnection, or partially by the separation mechanism described above, part of the free energy stored in the bulk plasma is ejected gradually, and system goes to a metastable state. The relaxed state has a more peaked pressure profile in the core than the initial state. Though we have not completed the comparison of this process with ELMs, it might be related to the experimental observation that the eruption occurs from the outboard side into the plasma. 
The authors would like to thank the NSTX team for providing us with the reconstruction data.

(Manuscript received Jan. 25, 2005,

revised April 6, 2005)

\section{References}

(1) A. Kirk, H.R. Wilson, G.F. Counsell, R. Akers, E. Arends, S.C. Cowley, J. Dowling, B. Lloyd, M. Price, M. Walsh, and MAST Team: "Spatial and Temporal Structure of EdgeLocalized Modes", Phys. Rev. Lett., Vol.92, 245002 (2004)

(2) J.L. Terry, S.J. Zweben, K. Hallatschek, B. LaBombard, R.J. Maqueda, B. Bai, C.J. Boswell, M. Greenwald, D. Kopon, W.M. Nevins, C.S. Pitcher, B.N. Rogers, D.P. Stotler, and X.Q. Xu: "Observations of the turbulence in the scrape-offlayer of Alcator C-Mod and comparisons with simulation", Phys. Plasmas, Vol.10, pp.1739-1747 (2004)

(3) J.A. Boedo, D.L. Rudakov, R.A. Moyer, G.R. McKee, R.J. Colchin, M.J. Schaffer, P.G. Stangeby, W.P. West, S.L. Allen, T.E. Evans, R.J. Fonck, E.M. Hollmann, S. Krasheninnikov, A.W. Leonard, W. Nevins, M.A. Mahdavi, G.D. Porter, G.R. Tynan, D.G. Whyte, and X. Xu: "Transport by intermittency in the boundary of the DIII-D tokamak", Phys. Plasmas, Vol.10, pp.1670-1677 (2004)

(4) O.E. Garcia, V. Naulin, A.H. Nielsen, and J. J. Rasmussen: "Computations of Intermittent Transport in Scrape-Off Layer Plasmas", Phys. Rev. Lett., Vol.92, p.165003 (2004)

(5) H.R. Wilson and S.C. Cowley: "Theory for Explosive Ideal Magnetohydrodynamic Instabilities in Plasmas", Phys. Rev. Lett., Vol.92, p.175006 (2004)

(6) T. Hayashi, N. Mizuguchi, H. Miura, R. Kanno, N. Nakajima, and M. Okamoto: "Nonlinear MHD Simulations of Spherical Tokamak and Helical Plasmas", IAEA-CN-94/TH/6-3, 19th IAEA Fusion Energy Conference, Lyon, France (2002)
Naoki Mizuguchi (Member) was born in Kyoto on July 2,

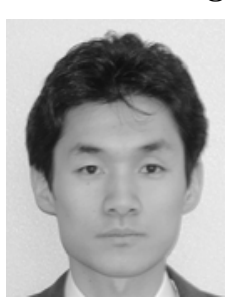
1972. He received a Ph.D. degree in engineering from Sokendai in 2000, and is presently a research associate at National Institute for Fusion Science. He has worked on nonlinear simulation research, and has been interested in visualizations.

Riaz Khan (Non-member) was born in Pakistan on Decem-

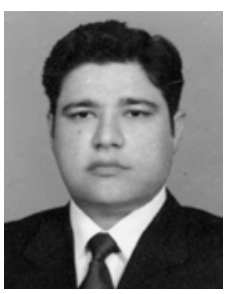
ber 15, 1976. He received two Master degrees, first one in Physics and second one in Nuclear Engineering from Quaid Azam University Pakistan, and is presently Ph.D. Student at Sokendai. He is working in the field of nonlinear plasma simulation.

Takaya Hayashi (Non-member) received a Ph.D. degree

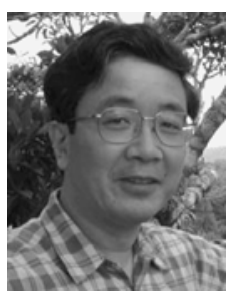
from University of Tokyo. He is presently a professor and the director of Theory and Data Analysis division, National Institute for $\mathrm{Fu}-$ sion Science. He has worked on nonlinear dynamics of plasma. 